\title{
Reemplazo del colorante rojo 40 por antocianinas en mermeladas; influencia en el color y las propiedades reológicas.
}

\author{
Replacement of the red dye 40 with anthocyanins in jams; influence on color and \\ rheological properties.
}
Almachi Villalba D.*1; Suárez Heredia M. ${ }^{2}$; Bonilla Valladares P. ${ }^{3}$; Díaz Basantes M. ${ }^{4}$
1,2,3 Universidad Central del Ecuador. Facultad de Ciencias Químicas.Carrera de Química email:dpalmachi@uce.edu.ec* email:masuarez@uce.edu.ec email:pmbonilla@uce.edu.ec
${ }^{5}$ Universidad Central del Ecuador. Facultad de Ciencias Químicas.Carrera Química de Alimentos email:mfdiaz@uce.edu.ec

\author{
Artículo original \\ Recibido: septiembre 2020 \\ DOI: https://doi.org/10.29166/quimica. \\ v7i1.2592 \\ Publicado: abril 2021
}

\begin{abstract}
Resumen: Se determinó el efecto sobre el color y las propiedades reológicas al reemplazar el colorante sintético rojo 40 por colorantes antociánico y copigmentos en una formulación de mermelada. Se preparó una base de mermelada con una metodología y fórmula unitaria estandarizadas. Se añadió los colorantes variando su concentración entre 200 y 800 ppm; es decir; los factores de estudio fueron el tipo de colorante y su concentración y las variables respuesta fueron el color y el área de histéresis. Se determinó que el tipo de colorante utilizado no presentó significancia estadística sobre el color, debido a que la interacción del copigmento en una matriz con $46,75 \%$ de agua desfavorece el equilibrio de las fuerzas de interacción entre la antocianina y el ácido rosmarínico, que forman el copigmento. El efecto de interacción generado por la concentración y tipo de colorante tiene significancia estadística en el área de histéresis. Por lo tanto, la reología permitió determinar que la adición de los colorantes aumenta el área de histéresis; es decir, favorece la destrucción del sistema al ser sometido a esfuerzos. Sin embargo, los colorantes podrían ser aplicados a nivel industrial, ya que no se observaron lazos de histéresis que significarían la destrucción irreversible del producto
\end{abstract}

Palabras clave: área de histéresis, propiedades reológicas, colorante rojo 40, colorante antociánico, copigmento

Abstract: The effect on color and rheological properties was determined by replacing red 40 synthetic dye with anthocyanin dye and copigments in a jam formulation. A jam base was prepared with a standardized methodology and unitary formula. The dyes were added varying their 
concentration between 200 and $800 \mathrm{ppm}$; that is, the study factors were the type of dye and its concentration and the response variables the color and the area of hysteresis. It was established that the type of dye used did not have statistical significance on color, because the interaction of the copigment in a matrix with $46.75 \%$ of water discourages the equilibrium of the interaction forces between the anthocyanin and the rosmarinic acid that form the copigment. Otherwise, the interaction effect generated by the concentration and type of dye has statistical significance in the hysteresis area. Thus, The Rheology allowed to determine that the addition of dyes increases the hysteresis area; in the other words, it favors the destruction of the system when subjected to efforts. However, it could have industrial applicability, since were not observed hysteresis ties, that would mean the irreversible destruction of the product.

Key Words: zone of hysteresis, rheological properties, dye red 40, anthocyanin dye, copigments

\section{Introducción}

El color de los alimentos es uno de los parámetros más importantes para los consumidores al momento de elegir un producto; los seres humanos tienen la tendencia a elegir alimentos con colores vivos ya que lo relacionan con frescura $y$ beneficios para su salud. ${ }^{1}$

La industria alimentaria utiliza colorantes, que en su mayoría son de origen sintético, debido a su alto poder de tinción. El uso de colorantes sintéticos genera daños a la salud, como lo demuestra el estudio desarrollado en la ciudad de Southampton. La investigación se enfocó en niños entre los 3 y 9 años, definió el efecto del consumo de ciertas mezclas de benzoato de sodio con colorantes; entre ellos el rojo 40, representado en la figura 1. El estudio concluyó que las mezclas podrían exacerbar la hiperactividad en los niños hasta la niñez media; motivando a organismos reguladores de Europa la prohibición de su consumo o que en la etiqueta se incluya la leyenda "podría tener un efecto en la actividad y atención de los niños". ${ }^{2}$

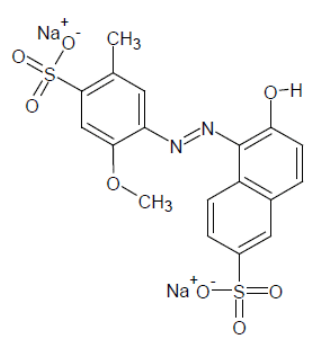

Figura 1 Estructura química de colorante sintético rojo 40 .

Se ha demostrado que la microbiota intestinal metaboliza el $70 \%$ de los colorantes azo como el rojo 40; a través, de enzimas azoreductasas; en dónde, los principales productos del metabolismo son compuestos químicos análogos al 1-naftol y aminas aromáticas tóxicas o mutagénicas. ${ }^{3}$ Por tanto, la industria alimenticia debe buscar nuevas alternativas; en donde, el uso de colorantes naturales debe cobrar relevancia, considerando los beneficios adicionales que éstos pueden aportar. Por ejemplo, la administración de una mezcla de bayas maduras de las variedades 'Tifblue' (Vaccinium virgatum asheii Aiton) y 'Rubel' (Vaccinium corymbosum L.) a adultos mayores con deterioro cognitivo leve; durante 16 semanas, mejora la memoria visual espacial. ${ }^{4}$ Además, existen estudios que demuestran que las 
antocianinas representadas en la figura 2; tienen actividad anticancerígena positiva al atacar la inflamación, el estrés oxidativo y las vías de señalización apoptótica. ${ }^{5}$

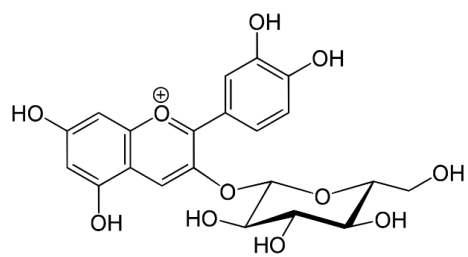

Figura 2 Estructura química de cianidina-3-Oglucósido.

Por lo tanto, la adición de antocianinas permite el desarrollo de alimentos funcionales; además, aumentan la vida útil y aceptabilidad de alimentos como; yogurt ${ }^{6}$, jarabes ${ }^{6}$ y caramelos duros, ${ }^{6}$ totillas de maíz. ${ }^{7}$ También se ha añadido antocianinas estabilizadas por copigmentación; a matrices alimenticias como; por ejemplo, néctares de fresa. ${ }^{8}$

Por otra parte, el tratamiento térmico empleado durante la elaboración de mermeladas provoca la pérdida de sustancias bioactivas, provocando cambios en su coloración; por ejemplo, la elaboración de mermeladas de fresa a $85^{\circ} \mathrm{C}$ disminuyó en 20,57\% las antocianinas monoméricas totales en comparación con la fruta; además, el producto es más oscuro ya que disminuyó en $25.49 \%$ el parámetro L* con respecto a la fruta. ${ }^{9}$ Por lo tanto, la adición de antocianinas permite recuperar el color y las sustancias bioactivas perdidas en la manufactura de mermeladas.

El análisis sensorial es el método óptimo para determinar la calidad de los alimentos; sin embargo, se requiere mucho tiempo y dinero para formar y capacitar jueces expertos que solamente son capaces de analizar de 6 a 8 muestras diarias debido a la fatiga del panelista. ${ }^{10}$ Por el contrario, la reología permite determinar cómo los alimentos fluyen o se deforman en función de parámetros medibles como viscosidad instantánea inicial, área de histéresis, módulo elástico, módulo viscoso, tixotropía y reopexia. ${ }^{11}$

\section{Parte experimental}

\subsection{Materiales y reactivos}

Las materias primas para la formulación de la mermelada fueron grado alimenticio (USP): Pectina (Ceanpectin $\left.{ }^{\circledR}\right)$, ácido cítrico anhidro, sorbato de potasio, benzoato de sodio, fructosa, rojo 40. Etanol al 96\% grado técnico y hexano grado analítico. El estándar ácido rosmarínico al 96\% (Sigma-Aldrich).

Con respecto a los equipos se utilizó una balanza analítica y de precisión con marcas Denver y Ohanus respectivamente. El Rotavapor marca J.P. Selecta, una tamizadora marca Gilson, fluidos supercríticos marca SCF GREEN TECHNOLOGY y estufas de convección forzada y natural marca brinder; permitieron estandarizar la muestra de mortiño Vaccinium floribundum Kunt y extracción del colorante antociánico. Los equipos que permitieron determinar las propiedades fisicoquímicas de las formulaciones de mermelada fueron: un potenciómetro marca Horiba, rehómetro marca Malvern Instru, Calorímetro diferencial de barrido marca TA, espectrofotómetro marca Cary 50 y finalmente los refractómetros de $0-32^{\circ}$ Brix y 50-80 ${ }^{\circ}$ Brix, marca BOECO. 


\subsection{Métodos}

\subsubsection{Estandarización de la muestra de mortiño.}

Para la estandarización de la muestra de mortiño Vaccinium floribundum Kunt, que consiste en la selección, lavado, desinfección, secado, molienda y desengrasado; se utilizó la metodología planteada en la investigación de Suárez \& Narváez (2016) ${ }^{12}$; se hizo una modificación en cuanto al desengrasado que se realizó con $\mathrm{CO}_{2}$ supercrítico en el equipo SCF GREEN TECHNOLOGY Speed SFE con los parámetros que se detallan en la tabla 1.

Tabla 1. Condiciones para el desengrasado con $\mathrm{CO}_{2}$ supercrítico

\begin{tabular}{|l|l|}
\hline Parámetro & Control de análisis \\
\hline Fluido supercrítico $\mathrm{CO}_{2}$ & 350,6 bar y $40^{\circ} \mathrm{C}$ \\
\hline Velocidad de entrada $\mathrm{CO}_{2}$ & $5 \mathrm{~L} / \mathrm{min}$ \\
\hline $\begin{array}{l}\text { Tanque de almacenamiento de la } \\
\text { materia prima }\end{array}$ & $42^{\circ} \mathrm{C}$ \\
\hline $\begin{array}{l}\text { Vial de recepción del material } \\
\text { apolar }\end{array}$ & $89^{\circ} \mathrm{C}$ \\
\hline
\end{tabular}

\subsubsection{Extracción de antocianinas.}

La muestra de mortiño Vaccinium floribundum Kunt estandarizada; se sometió a percolación con etanol al $96 \%$ a 23 gotas por minuto. Concluida la percolación; al extracto etanólico de antocianinas se le realizó 3 extracciones líquido-líquido con 50 $\mathrm{mL}$ de hexano. Se concentraron los extractos en rotavapor J.P. Selecta RS 3000V; los residuos se secaron a $40^{\circ} \mathrm{C} \pm 1{ }^{\circ} \mathrm{C}$ en la estufa con aire circulante BINDER BF53 y se controló hasta peso constante en balanza analítica Denver Pt 214. Con este extracto se prepararon disoluciones de concentración de $10 \mathrm{~g} / \mathrm{L}$ y $5 \mathrm{~g} / \mathrm{L}$ de antocianinas.

\subsubsection{Preparación de copigmentos.}

Se prepararon en etanol al 96\%. $5 \mathrm{~mL}$ de soluciones de $10 \mathrm{~g}$ de antocianinas/L y $960 \mathrm{mg}$ de ácido rosmarínico/L. Se mezclaron y la solución resultante se secó en una caja Petri prepesada hasta la evaporación total del etanol en una estufa con aire circulante BINDER BF53 a $40^{\circ} \mathrm{C} \pm 1{ }^{\circ} \mathrm{C}$. $\mathrm{La}$ evaporación total del etanol fue verificada por el peso constante.

\subsubsection{Caracterización espectrofotométrica del} extracto total de antocianinas y copigmentos

Se realizó un barrido en un rango entre 400-700nm de las soluciones etanólicas de $5 \mathrm{~g} / \mathrm{L}$ de antocianinas y copigmentos. Posteriormente se calculó el desplazamiento batocrómico e hipercrómico.

\subsubsection{Caracterización térmica del extracto total} de antocianinas y copigmentos.

Se utilizó el calorímetro diferencial de barrido TA Instruments Q2000 para obtener termogramas de extractos secos de antocianinas y copigmentos. Se utilizaron cápsulas de aluminio Standar Pan TA T1G1122 y T1G1122. Se pesó en balanza Meter Toledo GWP® B451386191 de apreciación $0,001 \mathrm{mg}$ la cápsula de referencia; la cápsula vacía 
y aproximadamente $0,8 \mathrm{mg}$ de la sustancia a analizar. El análisis se realizó en un intervalo de temperatura de $15-200^{\circ} \mathrm{C}$ a una velocidad de calentamiento de $10^{\circ} \mathrm{C} / \mathrm{min}$.

2.2.6 Formulación de la mermelada y base de mermelada.
Las fresas comerciales se lavaron con abundante agua potable, se cortaron los pedúnculos de las fresas y se licuaron para obtener la pulpa.

Se pesó en balanza analítica Denver Pt 214 los reactivos de la fórmula unitaria que se especifica en la tabla 2. Se trituró en un mortero el sorbato de sodio y el benzoato de sodio (mezcla 1) y se molió en mortero el ácido cítrico.

Tabla 2. Fórmula unitaria de la mermelada.

\begin{tabular}{|l|c|}
\hline INGREDIENTE & PORCENTAJE \\
\hline Fructosa & 28,0 \\
\hline Pulpa & 70.9 \\
\hline Estabilizante para preparaciones de fruta & 0,1 \\
\hline Ceanpectin ${ }^{\circledR}$ & 0,5 \\
\hline Sorbato de potasio & 0,04 \\
\hline Benzoato de sodio & 0,04 \\
\hline Ácido cítrico & 0,42 \\
\hline TOTAL & $\mathbf{1 0 0}$ \\
\hline
\end{tabular}

En la figura 3 se observa el montaje del sistema para la preparación de la mermelada. Se colocó el agua, fructosa y la mezcla 1 en un vaso de precipitación de $250 \mathrm{~mL}$. El sistema se sometió por 5 minutos a $300 \mathrm{rpm}$ de agitación y la plancha de calentamiento a $50{ }^{\circ} \mathrm{C}$. Manteniendo las mismas condiciones se añadió el ácido cítrico previamente molido. Se añadieron los gelificantes -Estabilizante para preparaciones de fruta- y - Ceampectin ${ }^{\circledR}$-se subió la agitación a 500 rpm durante 1 minuto y se sometió al sistema por 27 minutos a $300 \mathrm{rpm}$ de velocidad de agitación con la plancha de calentamiento a $300^{\circ} \mathrm{C}$.

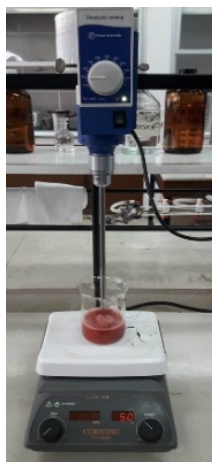

Figura 3. Montaje del sistema para la preparación de mermelada

Se pesó la mermelada en una balanza de precisión Ohanus PA 1502 y se determinó el rendimiento del proceso; considerando la fórmula unitaria como el $100 \%$. Al producto final también se midió el $\mathrm{pH}$ en el potenciómetro Horiba F12, los grados Brix $\left({ }^{\circ} \mathrm{Bx}\right)$ en el refractómetro de 50-80 ${ }^{\circ} \mathrm{Bx}$ BOECO $224159 \mathrm{CB}$ y finalmente se determinó la humedad. 
Para la preparación de la base de la mermelada, se consideró que la fresa posee $90 \%$ de agua ${ }^{13}$. Por lo tanto, se sustituyó la pulpa por $63,81 \%$ de agua tipo III; utilizando la metodología de la preparación de la mermelada mostrada en la tabla 2.

\subsubsection{Adición de colorantes.}

A la mermelada y la base de mermelada se añadieron los diferentes colorantes que se detalla en la tabla 3.

Tabla 3. Formulaciones con los respectivos colorantes

\begin{tabular}{|c|c|}
\hline Codificación & Descripción \\
\hline 200BA & $\begin{array}{l}\text { Base de mermelada con } 200 \text { ppm de extracto total de } \\
\text { antocianinas }\end{array}$ \\
\hline 200BAC & Base de mermelada con 200 ppm de copigmento \\
\hline 200BR40 & $\begin{array}{l}\text { Base de mermelada con } 200 \text { ppm de colorante } \\
\text { sintético rojo } 40\end{array}$ \\
\hline 800BA & $\begin{array}{l}\text { Base de mermelada con } 800 \mathrm{ppm} \text { de extracto total de } \\
\text { antocianinas }\end{array}$ \\
\hline 800BAC & Base de mermelada con 800 ppm de copigmento \\
\hline $200 \mathrm{MA}$ & $\begin{array}{l}\text { Mermelada de fresa con } 200 \mathrm{ppm} \text { de extracto total } \\
\text { de antocianinas }\end{array}$ \\
\hline 200MAC & Mermelada de fresa con $200 \mathrm{ppm}$ de copigmento \\
\hline 200MR40 & $\begin{array}{l}\text { Mermelada de fresa con } 200 \text { ppm de colorante } \\
\text { sintético rojo } 40\end{array}$ \\
\hline 800MA & $\begin{array}{l}\text { Mermelada de fresa con } 800 \mathrm{ppm} \text { de extracto total } \\
\text { de antocianinas }\end{array}$ \\
\hline 800MAC & Mermelada de fresa con 800 ppm de copigmento \\
\hline $800 \mathrm{MR} 40$ & $\begin{array}{l}\text { Mermelada de fresa con } 800 \mathrm{ppm} \text { de colorante } \\
\text { sintético rojo } 40\end{array}$ \\
\hline
\end{tabular}

\subsubsection{Estudio espectrofotométrico}

Se pesó en balanza analítica Denver Pt 214 0,1g de 200MA y se maceró en metanol por 1 hora. El macerado se filtró en membrana con tamaño de poro de $0,22 \mu \mathrm{m}$. Al filtrado se añadió $1,5 \mathrm{~mL}$ de estándar de antocianinas a $25 \mathrm{mg} / \mathrm{mL}$, se aforó a 10mL y se realizó un barrido en el rango del visible
$(400-700 \mathrm{~nm})$ en el espectrofotómetro UV-Vis Cary 50 ELOG103519.

El mismo procedimiento se desarrolló para 800MA, 200MAC, 800MAC, con la adición de su respectivo estándar. 
2.2.9 Evaluación de propiedades reológicas.

A la base de mermelada, y a las formulaciones 200BA, 200BAC, 800BA, 800BAC detalladas en

la tabla 5 , se les realizaron tres diferentes análisis en el rehómetro Bohlin 2004305 a un GAP de 2000 micra.

\subsubsection{Barrido de amplitud.}

Los parámetros de análisis se detallan en la tabla 4.

Tabla 4. Parámetros para el barrido de amplitud

\begin{tabular}{|l|l|}
\hline \multicolumn{1}{|c|}{ Parámetro } & Condición de análisis \\
\hline Frecuencia $(\mathrm{Hz})$ & 0,1 \\
\hline Mínimo estrés aplicado $(\mathrm{Pa})$ & 0,2387 \\
\hline Máximo estrés aplicado $(\mathrm{Pa})$ & 50 \\
\hline Aplicación de esfuerzos & Logarítmico \\
\hline Sentido & Ida y vuelta \\
\hline Tiempo de espera para el descenso de esfuerzos $(\mathrm{s})$ & 2 \\
\hline Tiempo de integración $(\mathrm{s})$ & 10 \\
\hline Periodos & 1 \\
\hline Número de puntos & 20 \\
\hline Tiempo por punto $(\mathrm{s})$ & 12 \\
\hline
\end{tabular}

Se graficó la curva $\mathrm{G}^{\prime \prime}$ y $\mathrm{G}^{\prime}$ en función de los esfuerzos.

\subsubsection{Barridos de frecuencia simple oscilatoria.}

Los parámetros de análisis que se detallan en la tabla 5.

Tabla 5. Parámetros para el barrido de frecuencia simple oscilatoria

\begin{tabular}{|l|l|}
\hline Parámetro & Condición de análisis \\
\hline Esfuerzo $(\mathrm{Pa})$ & 10 \\
\hline Mínima frecuencia $(\mathrm{Hz})$ & 0,1 \\
\hline Máxima frecuencia $(\mathrm{Hz})$ & 10 \\
\hline Aplicación frecuencias & Logarítmico \\
\hline Sentido & Ida y vuelta \\
\hline Tiempo de espera para el descenso de frecuencias (s) & 2 \\
\hline Periodos & 1 \\
\hline Número de puntos & 100 \\
\hline
\end{tabular}

Se graficó la curva $\mathrm{G}^{\prime \prime}$ y $\mathrm{G}^{\prime}$ en función de la frecuencia. 


\subsubsection{Viscosidad.}

Los parámetros de análisis que se detallan en la tabla 6.

Tabla 6. Parámetros para la viscosidad

\begin{tabular}{|l|l|}
\hline Parámetro & Condición de análisis \\
\hline Mínimo estrés aplicado $(\mathrm{Pa})$ & 0,2387 \\
\hline Máximo estrés aplicado $(\mathrm{Pa})$ & 10 \\
\hline Aplicación de esfuerzos & Lineal con análisis de Tixotropía \\
\hline Sentido & Ida y vuelta \\
\hline Tiempo de análisis $(\mathrm{s})$ & 10 \\
\hline Periodos & 1 \\
\hline Número de puntos & 50 \\
\hline Velocidad máxima $(\mathrm{rad} / \mathrm{s})$ & 10 \\
\hline
\end{tabular}

Se graficó la curva de viscosidad instantánea $\left(\eta_{\mathrm{i}}\right) \quad(+1,-1)$ y la concentración de colorante $\left(\mathrm{C}_{\mathrm{C}}\right)$ con en función de la velocidad de deformación $\left(\mathrm{s}^{-1}\right)$. dos niveles $(+1,-1)$; por lo tanto, se obtiene un Además, de la curva de flujo que es: Esfuerzo (Pa) diseño factorial completo $2^{2}$ a un intervalo del en función de la velocidad de deformación $\left(\mathrm{s}^{-1}\right)$. $\quad 95 \%$ de confianza y las variables respuesta fueron

\subsection{Diseño experimental} el área de histéresis (AH) y el color medio espectrofotométricamente

La tabla 7 detalla y codifica a los factores de estudio: Tipo de colorante $\left(\mathrm{T}_{\mathrm{C}}\right)$ con dos niveles

Tabla 7. Diseño experimental

\begin{tabular}{|c|c|c|}
\hline \multirow{2}{*}{ Factor } & \multicolumn{2}{|l|}{ Codificación } \\
\cline { 2 - 3 } & - & + \\
\hline Tipo de colorante $\left(T_{C}\right)$ & $\begin{array}{c}\text { Extracto total de } \\
\text { antocianinas }\end{array}$ & Copigmentos \\
\hline Concentración $(C)$ & $200 \mathrm{ppm}$ & $800 \mathrm{ppm}$ \\
\hline
\end{tabular}

\subsection{Análisis estadístico}

Para el diseño factorial completo se aleatorizaron las dos corridas experimentales en el programa estadístico JMP(C. Con los resultados de la primera réplica del diseño mediante Algoritmo de Yates se calculó para cada variable respuesta la magnitud y 
signo de los efectos primarios y del efecto de interacción.

Para la determinación de la significancia estadística de los efectos se registraron los resultados de las dos réplicas de diseño. Para cada tratamiento se obtuvo la diferencia (d) y se calculó la variancia estimada de acuerdo con la ecuación 1.

$$
\mathrm{S}_{\mathrm{i}}^{2}=\frac{\mathrm{d}^{2}}{2}
$$

Cálculo de la varianza estimada para cada tratamiento. ${ }^{14}$

La raíz cuadrada de la varianza estimada para cada tratamiento corresponde a la desviación estándar de la respuesta $S_{\text {resp }}$ que permitió calcular la desviación estándar de los efectos de acuerdo con la ecuación 2.

$$
\begin{gathered}
\mathrm{S}_{\text {efecto }}=\frac{2 \mathrm{~S}_{\text {resp }}}{\sqrt{\text { número de corridas efectivas }}} \\
\text { Cálculo de } \mathrm{S}_{\text {efecto }}{ }^{14}
\end{gathered}
$$

Se consideró si el efecto es estadísticamente significativo; mediante el criterio que se muestra en la ecuación 3 en donde $\mathrm{K}$ proviene de la distribución t de Student a un intervalo del 95\% de confianza. Procedimiento que permitió sugerir el uso de colorantes naturales en alimentos procesados.

$$
\mid \text { efecto } \mid>\mathrm{k} \mathrm{S}_{\text {efecto }}
$$

Criterio para la significancia estadística del efecto. $^{14}$

\section{Resultados y discusión}

\subsection{Extracción de la fase apolar}

Las condiciones de extracción de la fase apolar con $\mathrm{CO}_{2}$ supercrítico de una muestra estandarizada de mortiño Vaccinium floribundum Kunt permitieron extraer $1,01 \%$ de la fase apolar; que representa el $93,52 \%$, este cálculo se realizó en base al 1,00 \pm $0,8 \%$ de fase apolar reportado en la investigación desarrollada por Suárez \& Narváez (2016). ${ }^{12}$ Es decir, no se pudo extraer la totalidad de la fase apolar; esto podría deberse a que el tanque de almacenamiento de la materia prima se mantuvo a $40{ }^{\circ} \mathrm{C}$ para conservar los compuestos sensibles al calor; debido a que, en la investigación realizada por Carlotta Campalani, se determinó que el desengrasado óptimo del orujo de frutas con $\mathrm{CO}_{2}$ supercrítico es a una presión de 300 bar; un flujo de entrada del $\mathrm{CO}_{2}$ de $5 \mathrm{~mL} / \mathrm{min}$ y una temperatura de almacenamiento de la materia prima de $70^{\circ} \mathrm{C}$; pero, al disminuir la temperatura a $40^{\circ} \mathrm{C}$, disminuye la eficiencia de extracción en un $35 \%{ }^{15}$. Por lo tanto, se debe optimizar las condiciones para que mediante el uso de fluidos supercríticos se pueda extraer la totalidad de la fase apolar.

\subsection{Extracción de antocianinas}

Se determinó espectrofotométricamente que el extracto etanólico tiene un máximo de absorción de $538 \mathrm{~nm}$ que corresponde a la longitud de onda de absorción de las antocianinas.

Por otra parte; en la investigación de Catalina Vasco y colaboradores; se determinó por HPLC que el extracto de mortiño ecuatoriano Vaccinium floribundum Kunt contiene 345 mg cianidina-3-Oglucósido/100 g de muestra seca; además, proantocianidinas como $(+)$ catequina y (-) epicatequina en una concentración de $10 \pm 0.3$ y 8 $\pm 4.1 \mathrm{mg} / 100 \mathrm{~g}$ de muestra seca respectivamente ${ }^{16}$. 
3.3. Caracterización espectrofotométrica $\mathbf{y}$ calorimétrica del extracto total de antocianinas y copigmentos

El análisis espectrofotométrico de las soluciones etanólicas de $5 \mathrm{mg} / \mathrm{mL}$ de extracto total de antocianinas y copigmentos representados en la tabla 8, indican que existe un porcentaje de desplazamiento batocrómico del $0,17 \%$ y un porcentaje de desplazamiento hipercrómico de $2,25 \%$; es decir un aumento en la coloración

Adicionalmente, por calorimetría diferencial de barrido se demostró un desplazamiento de la temperatura inicial de degradación de $26,68^{\circ} \mathrm{C}$ de los copigmentos con respecto a las antocianinas; este cambio importante podría deberse a las interacciones $\Pi-\Pi$ intermoleculares entre las antocianinas y el copigmento (ácido rosmarínico $)^{17}$. Este resultado podría estar relacionado con la investigación de Nattapong Kanha y colaboradores; que compararon la cinética de degradación térmica de las antocianinas y sus respectivos copigmentos; se determinó que la copigmentación tiene un efecto positivo con respecto a las antocianinas; ya que, sube el tiempo de vida media ${ }^{18}$

Tabla 8. Caracterización espectrofotométrica y calorimétrica del extracto total de antocianinas y copigmentos

\begin{tabular}{|l|l|l|l|}
\hline \multirow{2}{*}{ Tipo de colorante } & \multicolumn{2}{|c|}{ Espectrofotometría } & Calorimetría diferencial de barrido \\
\cline { 2 - 4 } & $\begin{array}{l}\text { Máximo de } \\
\text { absorción }(\mathbf{n m})\end{array}$ & Absorbancia & $\begin{array}{l}\text { Temperatura inicial de degradación } \\
\left({ }^{\circ} \mathbf{C}\right)\end{array}$ \\
\hline $\begin{array}{l}\text { Extracto total de } \\
\text { antocianinas }\end{array}$ & 538 & 0,710 & 84,04 \\
\hline Copigmentos & 538,9 & 0,726 & 110,72 \\
\hline
\end{tabular}

\subsection{Elaboración de la mermelada y base de} mermelada

Es importante el orden de adición de las materias primas en el proceso de preparación de la mermelada y base de mermelada. La adición del ácido cítrico se hizo vez solubilizados el benzoato de sodio y el sorbato de potasio para asegurar la formación los ácidos sórbico y benzoico que tienen actividad antimicrobiana. ${ }^{19}$

La adición de los gelificantes -Ceanpectin ${ }^{\circledR}-\mathrm{y}-$ Estabilizante para preparaciones de fruta- debe hacerse una vez desarrollado el procedimiento anterior para evitar que en el sistema exista una competencia entre la precipitación y la gelificación. ${ }^{19}$

Otros aspectos considerados fueron el tiempo del tratamiento térmico y la velocidad de agitación. Un exceso del tratamiento térmico provocaría un daño irreversible al producto por la caramelización del azúcar. Por lo tanto, se incrementó la velocidad de agitación de $300 \mathrm{rpm}$ a $500 \mathrm{rpm}$ durante 1 minuto en la adición del -Ceampectin ${ }^{\circledR}$ - y el -Estabilizante para preparaciones de fruta- para evitar la formación de grumos en la solución y en la hélice del agitador. 
La estandarización del procedimiento en la preparación de mermeladas es importante porque permite la reproducibilidad de los resultados de la investigación; además, en la industria permite la correcta determinación de los costos de la carga fabril.

\subsection{Caracterización de la mermelada y base de mermelada}

Las características fisicoquímicas que posee la mermelada y la base de mermelada se representan en la tabla 9 y se puede observar que se encuentran en un pH óptimo de acuerdo con el (CODEX STAN 296-2009, MOD) para las confituras, jaleas y mermeladas. Las mermeladas se encuentran en un pH bajo debido a que el ácido evita la formación de ácidos pectínicos, con lo cual disminuyen las partículas cargadas y aumenta la tendencia de las moléculas a asociarse. ${ }^{20}$

Tabla 9. Propiedades fisicoquímicas de la mermelada y base de mermelada.

\begin{tabular}{|l|l|l|}
\hline Parámetro & Base de mermelada & Mermelada \\
\hline $\mathrm{pH}$ & 3,219 & 3,322 \\
\hline${ }^{\circ}$ Brix & 55 & 55 \\
\hline Humedad & $46,75 \% \pm 0,34 \%$ & $48,22 \% \pm 0,30 \%$ \\
\hline Rendimiento & $60,98 \% \pm 2,22 \%$ & $68,80 \% \pm 3,19 \%$ \\
\hline
\end{tabular}

El rendimiento del proceso presentado en la tabla 9; corresponde a todas las etapas del proceso, excepto a la adición del colorante. Este valor permite calcular la cantidad de colorante adecuada para la formulación final y estimar los costos de producción a escala industrial.

Además, las formulaciones tradicionales son elaboradas con $50 \%$ de sacarosa y tienen $65^{\circ}$ Brix; en cambio la mermelada desarrollada en esta investigación tiene como edulcorante fructosa en un $28 \%$ obteniéndose un producto con $10^{\circ}$ Brix menos. Esta característica proporciona una ventaja comparativa a la formulación elaborada y aporta a disminuir el consumo de azúcares en un producto que mantiene sus características sensoriales.

La base de mermelada y la mermelada tienen $55^{\circ}$ Brix, esto podría justificarse analizando que la mermelada supera en un $7.82 \%$ el rendimiento del proceso con respecto a la base de mermelada, es decir, en la preparación de la base de mermelada, aumentó la evaporación del agua, provocando un aumento en la concentración de sólidos totales y un menor rendimiento; posiblemente este aumento de la evaporación del agua no ocurrió en la preparación de la mermelada, por los componentes de la fruta.

\subsection{Estudio espectrofotométrico}

Los copigmentos tienen un desplazamiento hipercrómico del $2,25 \%$ con respecto de las antocianinas; es decir, un aumento de coloración. Sin embargo, como se representa en la tabla 10, la adición de 800ppm de antocianinas a la mermelada aportan una absorbancia de 0.033; y los 
copigmentos a la misma concentración; aportan una absorbancia de 0.034, es decir un desplazamiento hipercrómico del 3,03\%. Por lo tanto, la adición de copigmentos a la mermelada aumenta el desplazamiento hipercrómico, esto podría estar relacionado con el pH 3.219 de la mermelada; ya que, en la investigación de Nattapong Kanha se determinó que para los copigmentos de cianidina-3-O-glucósido con ácido ferúlico; el porcentaje de desplazamiento hipercrómico aumenta en $27,11 \%$, al subir de un pH 3 a un $\mathrm{pH}^{18}$.

Tabla 10. Estudio espectrofotométrico de las formulaciones

\begin{tabular}{|c|c|c|}
\hline Código & $\begin{array}{c}\text { Absorbancia en pico a } \\
\mathbf{5 3 8 n m}\end{array}$ & Variación de absorbancia \\
\hline \multicolumn{3}{|c|}{ MERMELADA CON ANTOCIANINAS } \\
\hline Estándar & 0,184 & 0,000 \\
\hline 000MA & 0,192 & 0,008 \\
\hline 200MA & 0,196 & 0,012 \\
\hline 800MA & 0,217 & 0,033 \\
\hline Estándar & 0,217 & 0,000 \\
\hline 000MAC & 0,219 & 0,002 \\
\hline 200MAC & 0,231 & 0,014 \\
\hline 800MAC & 0,251 & 0,034 \\
\hline
\end{tabular}

\subsection{Estudio reológico}

El estudio reológico se realizó con la base de mermelada sin la adición de colorantes. No se realizó el estudio reológico con la mermelada para evitar la introducción de variables que no se puedan controlar al añadir la fruta; ya que existen diferentes tipos de fresas. Estudios similares se desarrollan en la industria farmacéutica para evaluar la interacción de los excipientes con los principios activos de la formulación ${ }^{21}$

\subsubsection{Barrido de amplitud}

El barrido de amplitud de la base de mermelada representado en la figura 4 demuestra que en la base de mermelada no existe viscoelasticidad lineal porque no hay una tendencia constante del módulo elástico y viscoso en función del esfuerzo. ${ }^{22}$ Por el contrario, se observa una caída drástica del módulo viscoso en el intervalo de 0 a $10 \mathrm{~Pa}$, por lo tanto, este rango fue utilizado como intervalo de esfuerzos para los ensayos de viscosidad.

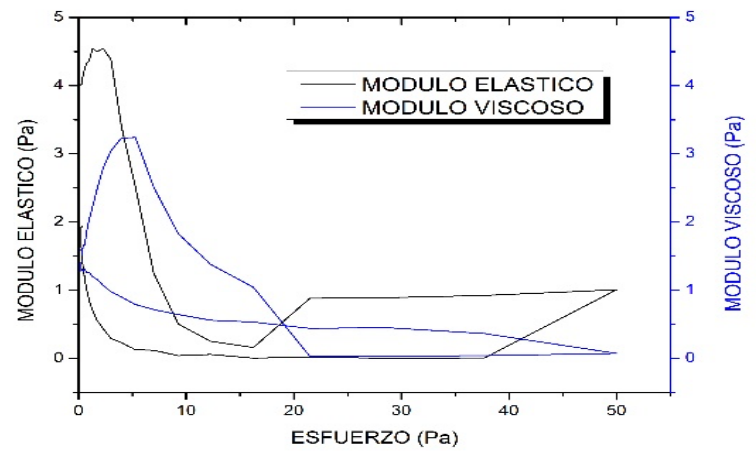

Figura 4. Barrido de amplitud de la base de mermelada 


\subsubsection{Barrido de frecuencia}

En el barrido de frecuencias de la base de mermelada representado en la figura 5 , se observa un aumento del módulo elástico a bajas frecuencias; es decir a largo plazo primará el módulo elástico sobre el viscoso en la base de mermelada.

Otro aspecto importante es que; la adición de los diferentes colorantes no modifica el barrido de amplitud y de frecuencias.

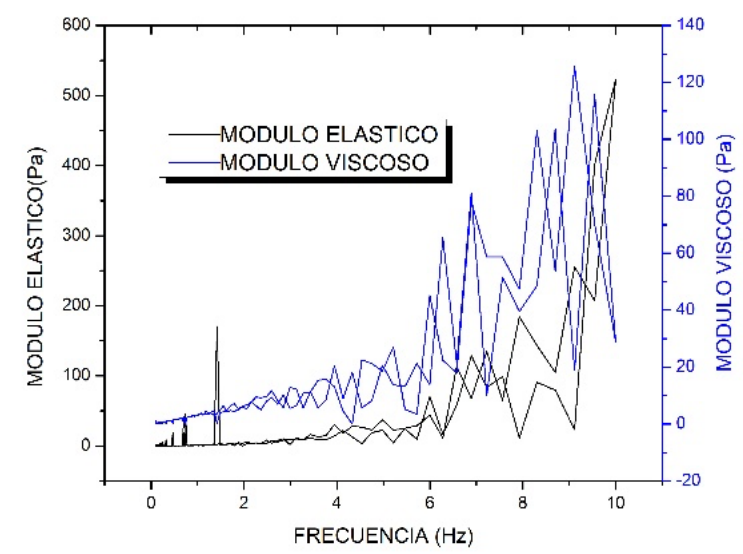

Figura 5. Barrido de frecuencias de la base de mermelada

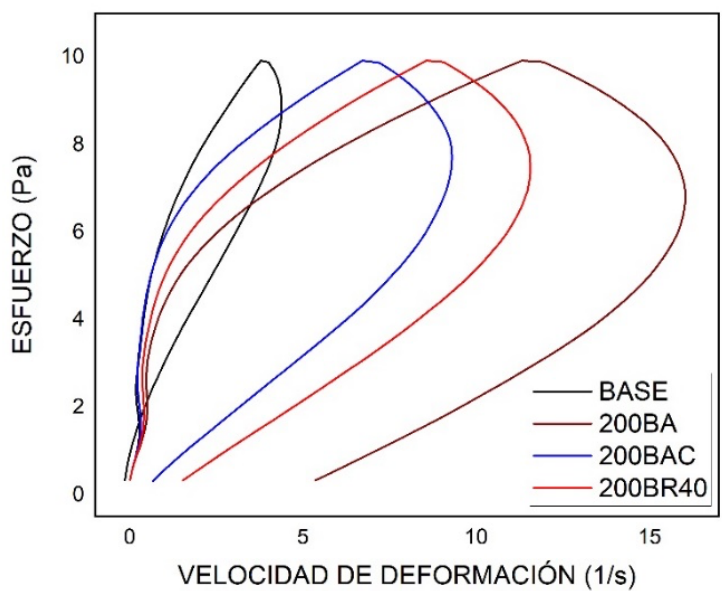

\subsubsection{Curvas de flujo}

En las curvas de flujo de la base de mermelada con los diferentes colorantes a $200 \mathrm{ppm}$ y $800 \mathrm{ppm}$ representadas en la figura 6 , se observa que la base de mermelada sin colorantes regresa al punto inicial del reograma; es decir, se reconstruye la estructura del sistema en la aplicación del descenso de los esfuerzos $(10,094 \mathrm{~s})$.

El fenómeno anterior puede deberse a que; la base de mermelada al ser sometida al ascenso de esfuerzos cortantes se va gradualmente rompiendo hasta que todas las partículas están dispersadas sol-, dando una viscosidad mínima. En el descenso de los esfuerzos las partículas comienzan a formar racimos, flóculos delgados y nuevamente el gel. ${ }^{23}$

Figura 6. Curvas de flujo de la base de mermelada con los diferentes colorantes: a 200 ppm (izquierda), 800 ppm (derecha) 
Por el contrario, las bases de mermelada con diferente concentración de colorantes no llegan al punto inicial del reograma; por lo tanto, necesitan más tiempo para reconstruir su estructura; sin embargo, no ocurrirán fenómenos de sedimentación y daños en el producto ya que en las curvas de flujo no se observan lazos de histéresis que son indicativos de la destrucción irreversible. ${ }^{23}$ El tiempo extra que necesitan las demás formulaciones para reconstruir su estructura en comparación con la base mermelada sin colorantes, se ve reflejado en las áreas de histéresis que se representan en la tabla 11, y son indicativos del grado de destrucción del sistema ${ }^{23}$.

Tabla 11. Áreas de histéresis de las formulaciones

\begin{tabular}{|c|c|}
\hline Tratamiento & Área de histéresis (Pa/s) \\
\hline BASE & 11,772 \\
\hline $200 \mathrm{BR} 40$ & 59,986 \\
\hline $200 \mathrm{BA}$ & 94,504 \\
\hline $200 \mathrm{BAC}$ & 46,509 \\
\hline $800 \mathrm{BR} 40$ & 91,315 \\
\hline $800 \mathrm{BA}$ & 80,342 \\
\hline $800 \mathrm{BAC}$ & 65,992 \\
\hline
\end{tabular}

La formulación 200BA aumenta el área de histéresis en $82,732 \mathrm{~Pa} / \mathrm{s}$ en comparación con la base de mermelada sin colorantes; esto podría deberse a que las antocianinas presentes en la base de mermelada provocan un cambio en la conformación de la pectina; es decir modifican su posición relativa y aumentan el grado de destrucción. ${ }^{24}$ Por el contrario, la formulación 200BAC aumenta el área de histéresis en 34,737 $\mathrm{Pa} / \mathrm{s}$ en comparación con la base de mermelada sin colorantes; es decir, las antocianinas favorecen el grado de destrucción con respecto a los copigmentos; esto podría deberse a que en los copigmentos las antocianinas forman enlaces de hidrógenos con el ácido rosmarínico; por lo tanto, ya no existe antocianina libre para provocar un cambio conformacional en la pectina.

Además, la formulación 800BR40 aumenta el área de histéresis en 79,543 Pa/s en comparación con la base de mermelada; esto podría suceder debido a que, como afirma Fereyra Susana (2015); ${ }^{20}$ la disminución de partículas cargadas en la mermelada aumenta la tendencia de las moléculas a asociarse. El rojo 40 representado en la figura 1; es una sal arildiazonio que aporta con cargas y disminuye la tendencia de las moléculas asociadas a formar el gel; por lo tanto; 800BR40 tiene un mayor grado de destrucción.

La formulación 800BAC aumenta el área de histéresis en $54,22 \mathrm{~Pa} / \mathrm{s}$ en comparación con la base de mermelada sin colorantes; esto podría deberse a que el ácido rosmarínico presente en la base de mermelada, no ayuda a disminuir el grado de destrucción; de igual manera la formulación 200BAC aumenta el área de histéresis en 34,737 $\mathrm{Pa} / \mathrm{s}$ en comparación con la base de mermelada.

Por lo tanto, la adición de antocianinas $\mathrm{y}$ copigmentos aumenta el área de histéresis; es decir 
el grado de destrucción del sistema. Sin embargo, podrían ser aplicados a nivel industrial porque no se observan lazos de histéresis que son indicativos del grado de destrucción del sistema coloidal ${ }^{23}$. De igual manera, en la investigación de VernonCarter; se determinó una disminución de la dureza de tortillas de maíz por la adición de antocianinas. ${ }^{7}$

\subsubsection{Curvas de viscosidad}

En la curva de viscosidad de la base de mermelada sin colorantes representada en la figura 8 , se observa un descenso de la viscosidad instantánea desde 24,13 Pas a 2,036 Pas cuando fluyen hasta una velocidad de desplazamiento de $4,377 \mathrm{~s}^{-1}$, es decir corresponde a un fluido pseudoplástico. Esta propiedad es importante porque la mermelada podrá formar pequeñas películas al ser untada en el pan o en el producto que el consumidor desee.

Además, se observa un efecto reopéxico al aplicar el descenso de los esfuerzos que corresponde a un aumento de la viscosidad instantánea de 17,33 Pas a $306 \mathrm{Pas}$ al descender los esfuerzos de 1,107 Pa a $0,9128 \mathrm{~Pa}$ respectivamente.

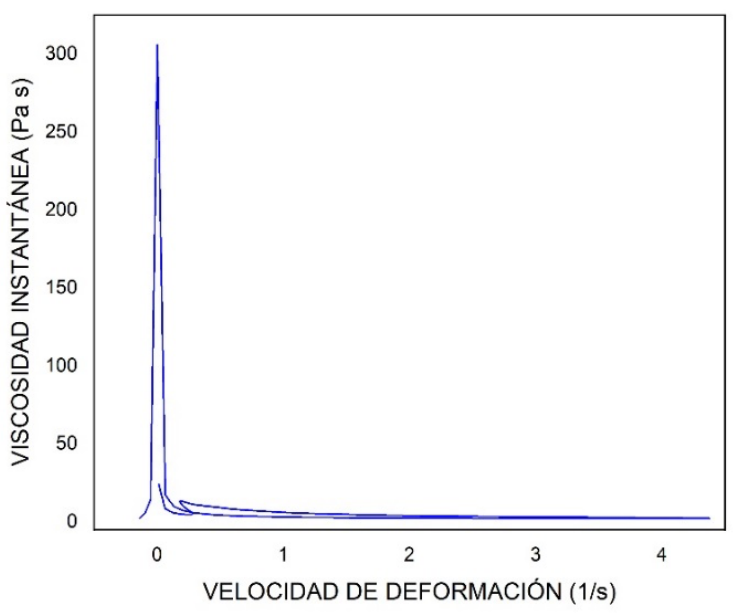

Figura 7. curva de viscosidad de la base de mermelada

Todos estos efectos, se comprenden mediante la teoría del entrecruzamiento de cadenas (Chain Entanglement), que explica el espesamiento de polímeros de alto peso molecular solubles en agua. Las cadenas son solvatadas por el agua formando enlaces de hidrógeno lo que les permite estirarse; el desorden así generado provoca un entrecruzamiento entre las cadenas atrapando componentes de la matriz. ${ }^{25}$ En el caso de la base de mermelada este efecto podría deberse a que los gelificantes -Ceampectin ${ }^{\circledR}-\mathrm{y}$ - Estabilizante para preparados de frutas- son polímeros de alto peso molecular y solubles en agua.
Por el contrario, como se observa en la figura 6, la adición de los colorantes en la base de mermelada a 200 ppm y 800 ppm baja el efecto reopéxico de la base de mermelada sin colorantes, esto podría justificarse asumiendo que la formación de enlaces de hidrógeno entre el agua y los polímeros disminuyen; debido a que los colorantes también forman enlaces de hidrógeno con los polímeros y/o las moléculas de agua. Por lo tanto, esta disminución representaría que las cadenas de polímero no se estiren y que no disminuya notablemente entrecruzamiento de estas. 

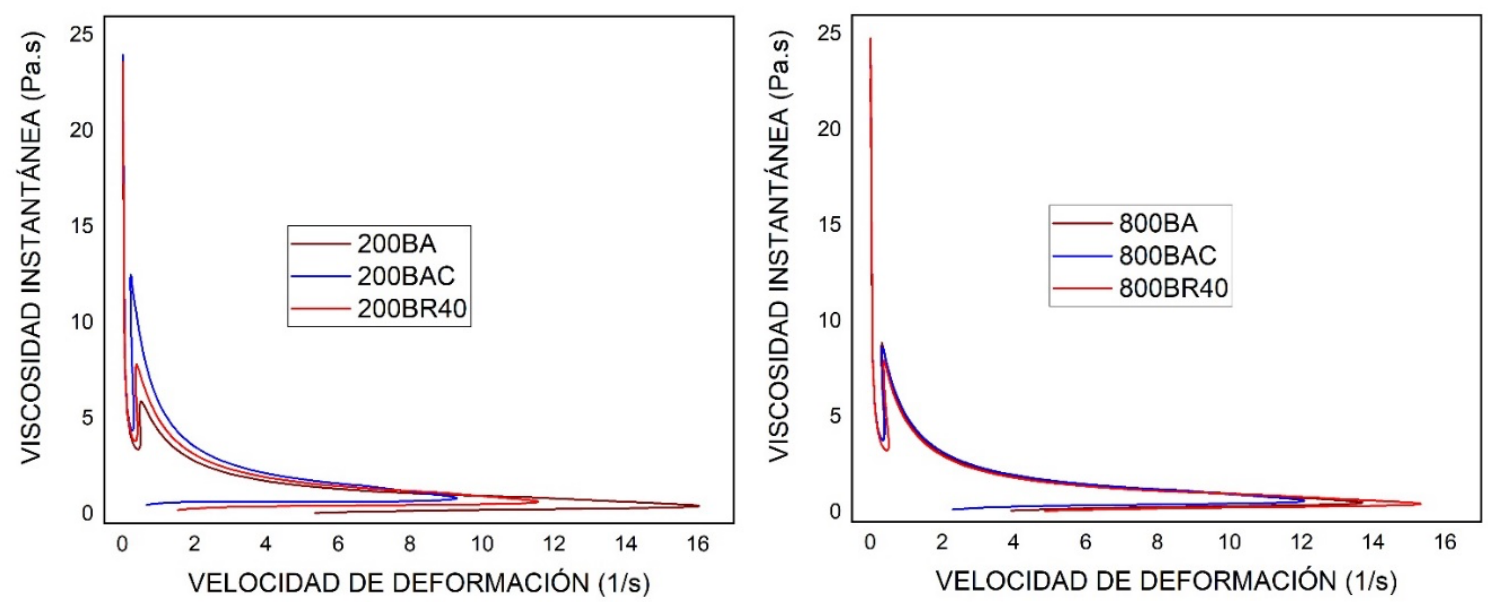

Figura 8. Curvas de viscosidad de la base de mermelada con los diferentes colorantes: a 200 ppm (izquierda), 800 ppm (derecha)

Las curvas de viscosidad permitieron determinar la viscosidad instantánea inicial; que es un parámetro indicativo de la presentación del producto. ${ }^{25} \mathrm{El}$ análisis de la tabla 12 muestra que en las bases de mermeladas con los tipos de colorantes a 200 ppm y 800ppm, no hay variaciones en la viscosidad instantánea inicial. Por lo tanto, las mermeladas con los diferentes colorantes a dichas concentraciones almacenadas en los envases presentarán la misma viscosidad

Tabla 12. Parámetros reológicos de las formulaciones

\begin{tabular}{|c|l|l|c|l|}
\hline Tratamiento & $\begin{array}{l}\text { Velocidad de } \\
\text { deslizamiento } \\
\text { final }\left(\mathbf{s}^{-\mathbf{1}}\right)\end{array}$ & Esfuerzo (Pa) & $\begin{array}{l}\text { Viscosidad } \\
\text { Instantánea } \\
\text { Inicial (Pas) }\end{array}$ & $\begin{array}{l}\text { Viscosidad } \\
\text { Instantánea } \\
\text { mínima (Pas) }\end{array}$ \\
\hline $200 \mathrm{BR} 40$ & 11,56 & 7,359 & 23,6 & 0,6365 \\
\hline $200 \mathrm{BA}$ & 16,04 & 6,744 & 22,22 & 0,4205 \\
\hline $200 \mathrm{BAC}$ & 9,3 & 7,734 & 23,98 & 0,8316 \\
\hline \multirow{2}{*}{$800 \mathrm{BR} 40$} & 15,33 & 6,939 & 24,78 & 0,4527 \\
\cline { 2 - 3 } & 15,33 & 6,744 & & 0,4398 \\
\hline $800 \mathrm{BA}$ & 13,71 & 6,938 & 22,67 & 0,5062 \\
\hline \multirow{2}{*}{$800 \mathrm{BAC}$} & 12,08 & 7,327 & 24,54 & 0,6067 \\
\cline { 2 - 3 } & 12,08 & 7,132 & & 0,5902 \\
\hline
\end{tabular}


Si la viscosidad instantánea de la mermelada es demasiado baja, se caerá de la rodaja de pan. ${ }^{22} \mathrm{Sin}$ embargo; no se pudieron realizar comparaciones con datos teóricos ya que en la bibliografía consultada no existen valores de la viscosidad de mermeladas en la cual ocurra este fenómeno.

Tomando como referencia el análisis anterior y la tabla 12; se concluye que las mejores formulaciones son 200BAC y 800BAC ya que tienen la mayor viscosidad instantánea mínima en comparación con las demás formulaciones.

\subsection{Análisis estadístico del color}

El diseño experimental para el color se desarrolló en la base de mermelada y la significancia estadística de los efectos representados en la tabla 13; fueron determinados por el método estadístico Algoritmo de Yates.

Tabla 13. Significancia estadística de los efectos para el área de histéresis

\begin{tabular}{|l|l|l|l|}
\hline Tipo de efecto & Variables & $\begin{array}{l}\text { Valor del efecto } \pm \text { error } \\
\text { estándar }\end{array}$ & $\begin{array}{l}\text { Significancia } \\
\text { estadística }\end{array}$ \\
\hline \multirow{2}{*}{ Primarios } & $\mathrm{T}_{\mathrm{c}}$ & $-0,0014 \pm 0,0067$ & No Significativo \\
\cline { 2 - 4 } & $\mathrm{C}_{\mathrm{c}}$ & $0,0694 \pm 0,0064$ & Significativo \\
\hline Secundario & $\mathrm{T}_{\mathrm{c}} \cdot \mathrm{C}_{\mathrm{c}}$ & $-0,0034 \pm 0,0099$ & No Significativo \\
\hline
\end{tabular}

Tal como se esperaba la concentración del colorante tiene un efecto significativo en el color. Sin embargo, no existe significancia estadística entre los tipos de colorantes; por lo tanto, la adición de los copigmentos a la mermelada no generará una mayor coloración en comparación con las antocianinas. Esta determinación es importante ya que la producción de copigmentos implica el tratamiento de antocianinas con fenoles libres, lo que incrementa los costos del colorante; en consecuencia, el costo del producto final.
La ecuación 4 establece que la absorbancia -color, únicamente depende de la concentración de colorante en la zona experimental analizada.

$$
\mathrm{A}=0.0879-0,0347 \mathrm{C}_{\mathrm{c}}
$$

Ecuación matemática que describe la absorbancia en función del efecto significativo

\subsection{Análisis estadístico de las propiedades reológicas}

En la tabla 14 se representa la significancia estadística de los efectos para el área de histéresis, determinado por el método estadístico Algoritmo de Yates. 
Tabla 14. Significancia estadística de los efectos para el área de histéresis

\begin{tabular}{|l|c|l|l|}
\hline Tipo de efecto & Variables & $\begin{array}{l}\text { Valor del efecto } \pm \text { error } \\
\text { estándar }\end{array}$ & $\begin{array}{l}\text { Significancia } \\
\text { estadística }\end{array}$ \\
\hline Primarios & $\mathrm{T}_{\mathrm{c}}$ & $-31,1725 \pm 4,9362$ & Significativo \\
\cline { 2 - 4 } & $\mathrm{C}_{\mathrm{c}}$ & $2,6605 \pm 6,6124$ & No Significativo \\
\hline Secundario & $\mathrm{T}_{\mathrm{c}} \cdot \mathrm{C}_{\mathrm{c}}$ & $16,8225 \pm 3,5048$ & Significativo \\
\hline
\end{tabular}

Por lo tanto, el área de histéresis depende del tipo de colorante y de la interacción con la concentración de colorante en la zona experimental analizada, como se representa en la ecuación 5.

$$
\mathrm{AH}=71,8368-15,5863 \mathrm{~T}_{\mathrm{c}}+8,4113 \mathrm{~T}_{\mathrm{c}} \cdot \mathrm{C}_{\mathrm{c}}
$$

Ecuación matemática que describe el área de histéresis en función del efecto significativo

Cuando la interacción de los efectos es significativa, las conclusiones que se obtienen a partir de los efectos primarios no siempre son correctas. Por lo tanto, sólo se interpretan los efectos primarios de aquellos factores que no interactúan con ningún otro. ${ }^{21}$

En base análisis anterior y la tabla 14, se concluye que la interacción del tipo de colorante con la concentración tiene significancia estadística en el área de histéresis. Esto podría deberse a los cambios conformacionales, formación de enlaces de hidrógeno que se detallan en la sección 3.7. El enlace de hidrógeno es una atracción electrostática débil (20 kJ/mol o $4.7 \mathrm{kcal} / \mathrm{mol})$, en comparación con el enlace covalente $(400 \mathrm{~kJ} / \mathrm{mol}$ o $95 \mathrm{kcal} / \mathrm{mol})$; sin embargo, como todas las antocianinas y copigmentos tienen la capacidad de formar enlaces de hidrógeno, en conjunto representan una gran fuerza. ${ }^{27}$

\section{Conclusión}

La adición del colorante sintético rojo 40, antocianinas y copigmentos, influyeron en las propiedades reológicas de una formulación base de mermelada; esto se ve reflejado en el aumento del área de histéresis, sin embargo, los colorantes estudiados podrían ser aplicados a nivel industrial, ya que no se observan lazos de histéresis que son indicativos de la destrucción irreversible del sistema al someterse a esfuerzos. Con respecto al color, la adición de copigmentos a la mermelada, aumenta el efecto hipercrómico; pero no es estadísticamente significativo con respecto al color que proporcionan las antocianinas sin copigmentar. De tal manera las antocianinas corresponden a un colorante natural efectivo para reemplazar el colorante rojo 40; es decir el desarrollo de alimentos funcionales.

\section{Agradecimientos.}

Al Ministerio de Ambiente del Ecuador, contrato marco de acceso a recursos genéticos MAE-DNBCM-2017-0078

\section{Referencias}

1. Lee, S. M., Lee, K. T., Lee, S. H., \& Song, J. K. (2013). Origin of human colour preference for food. Journal of Food Engineering, 119(3), 508-515. 
https://doi.org/10.1016/j.jfoodeng.2013.06. $\underline{021}$

2. McCann, D., Barrett, A., Cooper, A., Crumpler, D., Dalen, L., Grimshaw, K., Kitchin, E., Lok, K., Porteous, L., Prince, E., Sonuga-Barke, E., Warner, J. O., \& Stevenson, J. (2007). Food additives and hyperactive behaviour in 3-year-old and 8/9year-old children in the community: a randomised, double-blinded, placebocontrolled trial. Lancet, 370(9598), 1560$1567 . \quad$ https://doi.org/10.1016/S01406736(07)61306-3

3. Chavez-Bravo, E., Alonso-Calderon, A. I. A., Sanchez-Calvario, L., CastanedaRoldan, E., Vidal Robles, E., \& SalazarRobles, G. (2016). Characterization of the degradation products from the red dye 40 by enterobacteria. Journal of Pure and Applied Microbiology, 10(4), 2569-2575. https://doi.org/10.22207/JPAM.10.4.12

4. Krikorian, R., Kalt, W., McDonald, J. E., Shidler, M. D., Summer, S. S., \& Stein, A. L. (2020). Cognitive performance in relation to urinary anthocyanins and their flavonoidbased products following blueberry supplementation in older adults at risk for dementia. Journal of Functional Foods, 64(July), 103667.

https://doi.org/10.1016/j.jff.2019.103667

5. Fakhri, S., Khodamorady, M., Naseri, M., Farzaei, M. H., \& Khan, H. (2020). The ameliorating effects of anthocyanins on the cross-linked signaling pathways of cancer dysregulated metabolism. Pharmacological Research,

104895. https://doi.org/10.1016/j.phrs.2020.104895

6. Swer, T. L., Chauhan, K., Mukhim, C., Bashir, K., \& Kumar, A. (2019). Application of anthocyanins extracted from Sohiong
(Prunus nepalensis L.) in food processing. Lwt, $\quad$ 114(July),

108360. https://doi.org/10.1016/j.lwt.2019.108360

7. Vernon-Carter, E. J., Alvarez-Ramirez, J., Bello-Perez, L. A., Gonzalez, M., Reyes, I., \& Alvarez-Poblano, L. (2020). Supplementing white maize masa with anthocyanins: Effects on masa rheology and on the in vitro digestibility and hardness of tortillas. Journal of Cereal Science, 91(September 2019), 102883. https://doi.org/10.1016/j.jcs.2019.102883

8. Ertan, K., Türkyılmaz, M., \& Özkan, M. (2020). Color and stability of anthocyanins in strawberry nectars containing various copigment sources and sweeteners. Food Chemistry, $\quad 310, \quad 125856$. https://doi.org/10.1016/j.foodchem.2019.12 5856

9. Martinsen, B. K., Aaby, K., \& Skrede, G. (2020). Effect of temperature on stability of anthocyanins, ascorbic acid and color in strawberry and raspberry jams. Food Chemistry, $\quad 316$, 126297. https://doi.org/10.1016/j.foodchem.2020.12 6297

10. Joyner, H. S. (2018). Explaining food texture through rheology. Current Opinion in Food Science, 21, 7-14. https://doi.org/10.1016/j.cofs.2018.04.003

11. Upadhyay, R., \& Chen, J. (2020). Rheology and tribology assessment of foods. In Biopolymer-Based Formulations. Elsevier Inc. https://doi.org/10.1016/b978-0-12816897-4.00028-X

12. Suárez, M., \& Narváez, G. (2016). Copigmentación intermolecular de antocianinas glicosiladas. Quito: editorial académica española.

13. P. Alvarado and D. Espinoza. (2013). 
Estudio investigativo de la frutila y su aplicacipon en la gastronomía.

14. Vega, R. (2016). Curso de diseño experimental y análisis estadístico. Quito, Ecuela Politécnica Nacional.

15. C. Campalani et al. (2020), "Supercritical $\mathrm{CO} 2$ as a green solvent for the circular economy: Extraction of fatty acids from fruit pomace," J. CO2 Util., vol. 41, no. July, p. 101259, doi: 10.1016/j.jcou.2020.101259.

16. C. Vasco, K. Riihinen, J. Ruales, and A. Kamal-Eldin. (2009). "Chemical composition and phenolic compound profile of mortiño (vaccinium floribundum kunth)," J. Agric. Food Chem., vol. 57, no. 18, pp. 8274-8281, doi: 10.1021/jf9013586.

17. Zhao, X., Ding, B. W., Qin, J. W., He, F., \& Duan, C. Q. (2020). Intermolecular copigmentation between five common 3-Omonoglucosidic anthocyanins and three phenolics in red wine model solutions: The influence of substituent pattern of anthocyanin B ring. Food Chemistry, 326(17), 126960 . https://doi.org/10.1016/j.foodchem.2020.12 6960

18. Kanha, N., Surawang, S., Pitchakarn, P., Regenstein, J. M., \& Laokuldilok, T. (2019). Copigmentation of cyanidin 3-O-glucoside with phenolics: Thermodynamic data and thermal stability. Food Bioscience, 30(November 2018), 100419. https://doi.org/10.1016/j.fbio.2019.100419

19. López, G. (21 de noviembre de 2017). Criterios para la formulación de productos alimenticios. (D. Almachi, Entrevistador) Quito, Pichincha, Ecuador.

20. Fereyra, S. (2015). Estudio de prefactibilidad. Producción de confituras con integración sociolaboral. San Raael Mendoza: Universidad Nacional del Cuyo.

21. Ramírez, R. (2013). Estudios de interacción fármaco-excipiente de antiinflamatorios no esteroideos formulados en matrices sol-gel por calorimetría diferenciasl de barrido. México D.F: Universidad Nacional Autónoma de México.

22. World of Rehology. (2017). Obtenido de Anton Pear GmbH.

23. Remington, A. (2003). Farmacia (Vol. 20). Bueno Aires: PANAMERICANA.

24. Sui, Xiaonan. Zhang, Yan. Jiang, Lianzhoou. Zhou, W. (2018). Anthocyanins in foods. In Antocyanins in food (Vol. 7, Issue 3 ). Elsevier. https://doi.org/10.1080/1040839760952720 7

25. Maestro, A. (2002). Reología de espesantes celulósicos para pinturas al agua. Barcelona: Universidad de Barcelona.

26. Gutiérrez, H., \& Salazar, R. d. (2008). Análisis y diseño de esperimentos (Segunda edición ed.). México D.F: McGRAW HILL/INTERAMERICANA.

27. Badui, S. (2006). Química de Alimentos (Cuarta ed.). México D.F: Pearson. 